University of South Carolina

Scholar Commons

10-1994

\title{
Automating Workflows for Service Provisioning: Integrating Al and Database Technologies
}

Munindar P. Singh

Michael N. Huhns

University of South Carolina - Columbia, huhns@sc.edu

Follow this and additional works at: https://scholarcommons.sc.edu/csce_facpub

Part of the Computer Engineering Commons

\section{Publication Info}

Postprint version. Published in IEEE Expert, Volume 9, Issue 5, 1994, pages 19-23.

http://ieeexplore.ieee.org/servlet/opac?punumber=64

(C) 1994 by the Institute of Electrical and Electronics Engineers (IEEE)

This Article is brought to you by the Computer Science and Engineering, Department of at Scholar Commons. It has been accepted for inclusion in Faculty Publications by an authorized administrator of Scholar Commons. For more information, please contact digres@mailbox.sc.edu. 


\title{
Automating Workflows for Service Provisioning: Integrating AI and Database Technologies
}

\author{
Munindar P. Singh and Michael N. Huhns \\ Microelectronics and Computer Technology Corporation \\ Information Systems Division \\ 3500 West Balcones Center Drive \\ Austin, TX, U.S.A. 78759-5398 \\ (512) 338-3431 or \{huhns,msingh\}@mcc.com
}

\begin{abstract}
Workflows are the structured activities that take place in information systems in typical business environments. These activities frequently involve several database systems, user interfaces, and application programs. Traditional database systems do not support workflows to any reasonable extent: usually human beings must intervene to ensure their proper execution. We have developed an architecture based on AI technology that automatically manages workflows. This architecture, which executes on top of a distributed computing environment, has been applied to automating service provisioning workflows; an implementation that operates on one such workflow has been developed. This work advances the Carnot Project's goal of developing technologies for integrating heterogeneous database systems. It is notable in its marriage of AI approaches with standard database techniques.
\end{abstract}

Keywords: Workflows; Distributed AI; Heterogeneous Information Systems 
A facility for workflow management is an important component of the Carnot Project at MCC, which seeks to develop a suite of technologies that enable the integration of heterogeneous information resources. Project deliverables include an environment for the development of complex multisystem applications that access information stored in preexisting heterogeneous systems and maintain consistency constraints across them $[5,8]$.

Briefly, workflows consist of tasks, appropriately structured $[2,9,10]$. A task is any unit of computation that performs some useful function in a system. The tasks that are of particular interest are database transactions, but other computations, e.g., those that generate visualizations, can be presented in the same framework.

Integrating preexisting systems is in general a harder problem than designing distributed systems afresh. Many systems, especially those based on older mainframe architectures, allow data to be accessed only through arcane interfaces of limited functionality. The systems and their interfaces cannot be easily modified, and our work assumes they cannot. This is because of two main reasons: (1) the complexity of the programming effort that would be required to achieve any modifications, and (2) the constraint that older applications continue to run as before, since they typically have a wide user base that relies heavily upon them. Thus, the integration must permit newly developed applications to coexist with previous applications.

The major goal of the Carnot Project is to create general principles and approaches for integration of heterogeneous information resources. The Carnot Project is distinguished from other database research projects not only in terms of its goals, but also in having a larger and more significant AI component than is perhaps typical. We also undertake various application partnerships with our sponsors in order to develop prototype systems that address their specific problems. This not only serves to test our research ideas, but also suggests important research problems to work on. We did one such application partnership with one of our clients, a telecommunications 
company. In this paper, we describe the key ideas of our ongoing research, as well as how they were applied to the problems of this client. In Section 1, we describe how workflows and AI fit into heterogeneous information systems. In Section 2, we describe the specific problem we addressed and, in Section 3, our solution to it.

\section{Background}

Classical transaction processing in databases deals with executing access and update tasks on a single database. Such tasks are traditionally assumed to have the socalled ACID properties, which help simplify transaction management considerably [see sidebar on ACID properties]. However, they prove to be overly restrictive in loosely coupled heterogeneous environments. For example, one of the ways in which ACID tasks may be coordinated is through mutual commit protocols, which ensure that either all of a given set of tasks commit or none do. Such protocols, the classical example of which is the two-phase commit protocol, are notoriously inefficient when executed over networks. Further, to execute such a protocol, one requires access to the internal states of transactions, such as their precommit states. A transaction is in its precommit state when it is internally ready to commit, but is awaiting permission from the transaction manager to do so. Most commercial database systems do not provide access to such internal states, thereby making direct implementations of commit protocols extremely difficult.

The ACID properties are naturally realized when the correctness of database transactions is characterized through some purely syntactic or structural criterion, such as serializability [3]. However, serializability cannot be efficiently implemented in distributed systems whose component systems are autonomous. Instead of attempting to specify correctness criteria purely syntactically, we follow [7] and characterize them semantically. This allows us to specialize the correctness criteria to the given application at the cost of building a deeper model of the application domain. This helps 


\section{The ACID Properties}

Database transactions are designed to have the so-called ACID properties. However, these properties apply to tasks in general.

- Atomicity means that either all changes to the system state caused by a task happen or none do.

- Consistency means that a task takes the database from a consistent state to a consistent state.

- Isolation means that the intermediate results of a task are not visible to another task.

- Durability means that the changes committed by a task are persistent.

Jim Gray and Andreas Reuter, Transaction Processing: Concepts and Techniques, Morgan Kaufmann, San Mateo, CA, 1993. 
simplify several coordination requirements. For example, instead of executing mutual commit protocols, we can optimistically commit different tasks. If this action should prove erroneous, we undo the effects of incorrectly committed tasks. This is achieved by means of compensating transactions, whose definition depends on the semantics of the underlying domain.

Consequently, in heterogeneous environments, the unit of relevant activity is not a single database transaction, but rather a workflow that executes over a set of database and information resources. The constituent tasks of a workflow may be individually ACID, but the overall workflow usually is not. The problem is to ensure that no semantic constraint of the information model is violated despite this.

The activities that comprise a workflow of interest are typically already being carried out in the given organization. However, they are usually carried out by hand, with people intervening in several crucial stages to ensure that the necessary tasks are done and that organization-wide consistency constraints are enforced. The semantics that we alluded to above is supplied by the people or is implicitly encoded in different business procedures. The canonical examples of workflows are document flows through organizations. For instance, when an order is received, it must be entered into the system and several decisions must be taken to process it properly. These decisions would typically involve access to several information resources within an enterprise and possibly some outside of it. For example, a request to transfer money from one account to another requires that the authorization be verified, the account numbers be validated, and the source account be tested to have the required balance. External sources of information would be accessed for other requests, such as loan applications, where a credit bureau's databases may be consulted to determine the credit worthiness of an applicant.

It is of great importance to be able to handle the myriad error conditions that may arise in different workflows. The exception conditions in workflows are the ones 
that are the hardest to automate. It is in identifying and resolving such conditions and managing control and data flow appropriately that AI technology can contribute substantially.

\section{The Problem: Workflows for Service Provision- ing}

One of our clients provides a variety of telecommunication services. We studied the workflow for provisioning one such service that establishes a telecommunication link between two specified points. In the extant workflow, a set of paper forms is received that gives a number of relevant details about the service being ordered. These forms are entered into the system. A test is then performed to determine if certain essential telecommunication equipment is already in place. If it is, the service can be provided relatively quickly; otherwise, the processing must be delayed until the equipment is added.

Service provisioning typically takes several weeks and requires coordination among many operation-support systems and network elements. Configuring the operationsupport systems so that they can perform such a task often takes several months to complete. This proves to be of competitive significance in the business environment in which our client operates. Many of its competitors were formed in the last decade or so. Unlike our client, these companies are not encumbered with legacy systems and typically have more modern computational facilities.

We investigated ways to improve the provisioning of one type of communication facility - digital services. This provisioning takes more than two weeks and involves 48 separate operations - 23 of which are manual - against 16 different database systems. Our goals were to reduce this time to less than two hours and to provide a way in which new services could be introduced more easily. Our strategy for accomplishing these goals was to (1) interconnect and interoperate among the previously indepen- 
Figure 1: Abbreviated Semantic Model of the Provisioning Environment

dent systems, (2) replace serial operations by parallel ones by making appropriate use of relaxed transaction processing $[4,6,1]$, and (3) automate previously manual operations, thereby reducing the incidence of errors and delays.

An important goal of our project was to exhibit the feasibility of a workflow management approach that applies to workflows in general. Our specific challenge was to automate the provisioning workflow as a test-case to achieve the efficiencies of our client's competitors without discarding its legacy systems. We should note, however, our implementation is not meant at this stage for production use, but as a proof-of-concept exercise.

Figure 1 presents an entity-relationship diagram that shows the most relevant components of the semantic model of the provisioning problem. Figure 2 presents 
the basic structure of the workflow we studied - it shows the admissible executions when everything works correctly. Each node denotes a task. The partial order reflects the dependencies among the different tasks. Tasks cannot be initiated until all their dependencies are met; ordinarily, they must be initiated if those dependencies are satisfied.

Figure 2: The Provisioning Workflow Automated-only the default workflow is shown, without any exception paths 
Figure 3: A Distributed AI System for Workflow Management

\section{The Carnot Solution}

We defined a distributed agent architecture, shown in Figure 3, for intelligent workflow management that functions on top of Carnot's distributed execution environment. An important constraint on our design was to use existing procedures as much as possible so as to ensure that other applications were not adversely affected by our system. This turned out to be easily accommodated by our architecture; indeed, we welcomed not having to worry about the details of the mainframe systems on which we ran various tasks. Since the actual applications executed by the workflow were assumed to be defined already, our goal was to manage the overall structure of the applications in as domain-independent a manner as possible.

Our multiagent system consists of four agents that interact to produce the desired 
behavior. Figure 3 shows the key components of our architecture. The databases mentioned on the figure are assumed to include the relevant data and application programs that execute on them. The necessary applications are executed by the schedule processing agent; the user interface agent queries the systems to help a user fill in an order form completely and correctly, and to provide feedback about progress. This enables the detection of data inconsistencies. It is highly desirable to resolve inconsistencies early in the process.

The present architecture is enabled by our previous integration of an expert system shell, which has forward and backward chaining capabilities, a type system, and truth maintenance, into Carnot's distributed execution environment. This environment provides the basic message passing facility that our agents use to interact with other agents anywhere on the network. We used this facility to implement a scheme by which agents can exchange assertions, thereby triggering or disabling rules in each other. We augmented our scheme so that agents that are not expert systems can also participate in interactions, provided they satisfy a simple protocol. This enabled us to integrate transparently a graphical interaction agent, which is not an expert system shell, into the multiagent system.

Figure 4 describes our implementation at a high-level as an entity-relationship diagram. A key point to note is that the different tasks that correspond to the nodes of Figure 2 are modeled as database transactions. Each such transaction is initiated by an agent. Each task has associated with it a message type. The message type essentially encodes the computation that the underlying IMS databases must execute. When an agent executes a task, it does so by passing along the relevant message, i.e., the name of the file that contains it.

The agents operate as follows. The graphical-interaction agent helps a user fill in an order form correctly and completely, and checks inventories to give the user an estimate of when the order will be completed. It also informs the user about 
Figure 4: Description of the Transaction-Scheduling Agent's Implementation the progress of the order. The scheduling agent constructs the initial schedule for the given request, doing so on the assumption that the relevant subtasks will succeed. The tasks are scheduled with the maximum concurrency possible, while still satisfying all required precedence constraints.

The schedule processing agent executes the schedule by invoking different tasks as necessary. It maintains connections to the databases involved in telecommunication provisioning, and implements transactions on them. The schedule processor also ensures that different workflows do not interact spuriously. This is akin to the problem of concurrency control in traditional database systems. Concurrency control has to do with ensuring that different transactions that access the same data items do not access them in relative orders for which there are no equivalent serial executions. With a workflow, we need to ensure that subtasks on each database can be serialized in the same order. This may require delaying, or aborting and retrying, different subtasks. 
Figure 5: Conceptual Model for Schedule-Repairing Agent

If the schedule processor encounters an unexpected condition, e.g., the failure of a task, it notifies the scheduling agent, which communicates with the schedule repairing agent for advice on how to fix the problem. The advice can be information on how to restart a transaction, how to abort a transaction, how to compensate for a previously committed transaction, or how to clean-up a failed transaction. These actions are meant to restore semantic consistency across the system. For example, if the system is unable to allocate a span to a given service request, it aborts the entire request. The billing task, if already committed, is compensated. On the other hand, if the billing task fails, while the span allocation succeeds, the service order is allowed to proceed and the billing task is retried later. This example highlights the distinction between vital and nonvital subtasks. The failure of a vital subtask propagates to the global task; nonvital tasks can simply be retried. A conceptual model for the knowledge of the schedule-repairing agent is shown in Figure 5.

In our approach, the initial schedule is constructed on the assumption that things will succeed as expected. This leads to a small, easily executable, schedule. If error conditions should arise, they are accommodated at run-time by repairing the ini- 
tial schedule as appropriate. Some of this is automatic, since the undesirable and unexecuted parts of the schedule are disabled by the truth maintenance system of the scheduling agent when their preconditions fail to hold. [See sidebar on truth maintenance.]

The basic structure of this system is domain-independent. The details of the messages are clearly domain-dependent. Certain parameters, e.g., the identifier of the service request, are known to the scheduler, but most of the data is passed through the file system. The files are uniquely named using the known identifier, thereby allowing different requests to execute concurrently. The other domain-dependent components of the system are the procedures required to convert data formats from those produced by one application to those expected by the next. These translation routines were written using the tools Lex and Yacc. They are invoked as necessary by the schedule-processing agent. The remaining domain-dependent aspect of the provisioning workflow is in the resource constraints, which guide the scheduling and repairing processes.

\section{Conclusions}

We have completed a prototype implementation, which we treat as a proof-of-concept exercise, rather than a deployable system. The prototype is being reimplemented for installation in a restricted production environment (one switching center). If it is successful, it will be deployed in all switching centers by our client.

Certain desired features will call for AI technology in the final implementation: these include schedule repair and other semantical aspects of the domain. Because of business constraints, we do not expect to use our present Lisp-based system for these, although the ideas will be reimplemented in a $\mathrm{C}++$ or Rosette-based constraint processor. Certain other features, notably those to do with schedule processing, do not really require AI approaches, even though AI approaches are useful for rapidly 


\section{Truth Maintenance Systems in Workflow Scheduling}

A truth maintenance system (TMS) provides a simple, built-in, generic way of managing dependencies, such as in a workflow schedule. Justification-based TMSs assign a belief status of IN or OUT to each represented assertion. IN means believed and OUT means not believed. A justification for an assertion is a pair of lists of assertions: the IN-list and the OUT-list. A justification is valid if and only if all the assertions on its IN-list are IN and all the assertions on its OUT-list are OUT. An assertion must be labeled IN if it has at least one valid justification; otherwise, it must be labeled OUT.

TMSs simplify workflow scheduling. For example, the billing subtask proceeds on the assumption that the global task will not abort. Further, the billing task is retried on failure, but only if the global task does not abort in the meanwhile. The failure of the local circuit assignment subtask causes the global task to abort, thus removing the justification for proceeding with the billing and, if it already happened, adding the justification for proceeding with its compensation. Consequently, complicated but correct executions, such as when the billing subtask succeeds on the fifth attempt, the local circuit subtask fails, and the billing is canceled, can be realized even though they would not be explicitly specified.

Elaine Rich and Kevin Knight, Artificial Intelligence, McGraw Hill, 1991.

Michael N. Huhns and David M. Bridgeland, "Multiagent Truth Maintenance," IEEE Transactions on Systems, Man, and Cybernetics, vol. 21, no. 6, pp. 14371445, December 1991. 
prototyping them.

An alternative approach for scheduling tasks is to use operations research (OR) techniques, such as MRP II. With this approach, however, it is difficult to handle contingencies, such as the failure of a task. An OR approach would require new constraints to be added that reflect the failure, and then the MRP II system would have to be rerun to generate a new schedule. The new schedule might be quite different from the original one, which might cause additional problems, especially if the original schedule were already being executed.

It is safe to conclude that AI technology helped us sort out various issues and easily build a working system that could be tested. Having an implementation helps us understand the needed components and the interfaces among them. This aids in the design and testing of industrial-strength modules.

The benefits realized from automatic workflow processing include

- Improved turnaround time.

- Error checking of the initial input; validation of fields with respect to other fields and information in customer databases.

- Streamlining of the present procedures by removing redundant data gathering and processing.

- Ability to modify the structure of the overall procedure easily.

We believe that as information systems become more complex, there will be an increasing demand for AI technologies to manage them. It is likely, however, that AI technologies will have to take a somewhat different, possibly more mundane, form in applications, than might have been envisioned by the people who developed them. 


\section{References}

[1] Mansoor Ansari, Marek Rusinkiewicz, Linda Ness, and Amit Sheth, "Executing Multidatabase Transactions," Proceedings 25th Hawaii Int'l. Conf. on Systems Sciences, January 1992.

[2] Paul C. Attie, Munindar P. Singh, Amit P. Sheth, and Marek Rusinkiewicz, "Specifying and Enforcing Intertask Dependencies," Proceedings of the 19th VLDB Conference, 1993.

[3] Philip A. Bernstein, Vassos Hadzilacos, and Nathan Goodman, Concurrency Control and Recovery in Database Systems, Addison Wesley, 1987.

[4] Omran A. Bukhres, Jiansan Chen, Weimin Du, Ahmed K. Elmagarmid, and Robert Pezzoli, "InterBase: An Execution Environment for Heterogeneous Software Systems," IEEE Computer, Vol. 26, No. 8, Aug. 1993, pp. 57-69.

[5] Philip E. Cannata, "The Irresistible Move towards Interoperable Database Systems," First International Workshop on Interoperability in Multidatabase Systems, Kyoto, Japan, April 1991.

[6] Ahmed K. Elmagarmid, ed., Database Transaction Models for Advanced Applications, Morgan Kaufmann, San Mateo, CA, 1992.

[7] Hector Garcia-Molina and K. Salem, "Sagas," Proceedings of ACM SIGMOD Conference on Management of Data, 1987.

[8] Michael N. Huhns, Nigel Jacobs, Tomasz Ksiezyk, Wei-Min Shen, Munindar Singh, and Philip Cannata, "Integrating Enterprise Information Models in Carnot," International Conference on Intelligent and Cooperative Information Systems (ICICIS), Rotterdam, The Netherlands, June 1993, pp. 32-42. 
[9] Christine Tomlinson, Paul Attie, Philip Cannata, Greg Meredith, Amit Sheth, Munindar Singh, and Darrell Woelk, "Workflow Support in Carnot," IEEE Data Engineering, Vol. 16, No. 2, June 1993, pp. 33-36.

[10] Darrell Woelk, Paul Attie, Philip Cannata, Greg Meredith, Munindar Singh, and Christine Tomlinson, "Task Scheduling Using Intertask Dependencies in Carnot," ACM SIGMOD, 1993. 


\section{Biographical Sketches}

Michael N. Huhns received the B.S.E.E. degree in 1969 from the University of Michigan, Ann Arbor, and the M.S. and Ph.D. degrees in electrical engineering in 1971 and 1975, respectively, from the University of Southern California, Los Angeles.

He is a Senior Member of the Information Systems Division at the Microelectronics and Computer Technology Corporation, where he has been conducting research on the Argo, Antares, Reasoning Architectures, and Carnot projects. Prior to joining MCC in 1985, he was an Associate Professor of Electrical and Computer Engineering at the University of South Carolina, where he also directed the Center for Machine Intelligence. He was previously a Radar Systems Engineer at Hughes Aircraft Company.

Dr. Huhns is a member of Sigma Xi, Tau Beta Pi, Eta Kappa Nu, ACM, IEEE, and AAAI. He is the author of over 100 technical papers in machine intelligence and information systems, and an editor of the books Distributed Artificial Intelligence, Volumes I and II. His research interests are in the areas of distributed artificial intelligence, machine learning, enterprise modeling and integration, and computer vision.

Munindar P. Singh received the B.Tech. degree in computer science and engineering in 1986 from the Indian Institute of Technology, Delhi. He obtained the M.S. and Ph.D. degrees in computer science in 1988 and 1992, respectively, from the University of Texas, Austin. He is a Member of the Technical Staff in the Information Systems Division at MCC, where he has been conducting research on distributed artificial intelligence, heterogeneous database systems, and relaxed transaction processing. Dr. Singh has written a number of research papers in the above areas. His monograph on characterizing computational agents, entitled Multiagent Systems: A Theoretical Framework for Intentions, Know-How, and Communications, is being published by Springer Verlag. 